\title{
INTERACTION-POWERED SUPERNOVAE: RISE-TIME VERSUS PEAK-LUMINOSITY CORRELATION AND THE SHOCK-BREAKOUT VELOCITY
}

\author{
Eran O. OfeK ${ }^{1}$, Iair Arcavi ${ }^{1}$, David Tal $^{1}$, Mark Sullivan $^{2}$, Avishay Gal-Yam $^{1}$, Shrinivas R. Kulkarni $^{3}$, \\ Peter E. Nugent ${ }^{4,5}$, Sagi Ben-Ami ${ }^{1}$, David Bersier ${ }^{6}$, Yi CaO $^{3}$, S. Bradley Cenko ${ }^{7}$, Annalisa De Cia ${ }^{1}$, \\ Alexei V. Filippenko ${ }^{5}$, Claes Fransson ${ }^{8}$, Mansi M. Kasliwal ${ }^{9}$, Russ Laher ${ }^{10}$, \\ JASON Surace ${ }^{10}$, Robert QuimbY ${ }^{11}$, AND OFER YARON ${ }^{1}$ \\ ${ }^{1}$ Benoziyo Center for Astrophysics, Weizmann Institute of Science, 76100 Rehovot, Israel \\ ${ }^{2}$ School of Physics and Astronomy, University of Southampton, Southampton SO17 1BJ, UK \\ ${ }^{3}$ Cahill Center for Astronomy and Astrophysics, California Institute of Technology, Pasadena, CA 91125, USA \\ ${ }^{4}$ Computational Cosmology Center, Lawrence Berkeley National Laboratory, 1 Cyclotron Road, Berkeley, CA 94720, USA \\ ${ }^{5}$ Department of Astronomy, University of California, Berkeley, CA 94720-3411, USA \\ ${ }^{6}$ Astrophysics Research Institute, Liverpool John Moores University, Liverpool L3 5RF, UK \\ ${ }^{7}$ Astrophysics Science Division, NASA Goddard Space Flight Center, Mail Code 661, Greenbelt, MD 20771, USA \\ 8 Department of Astronomy, The Oskar Klein Centre, Stockholm University, AlbaNova University Centre, SE-106 91 Stockholm, Sweden \\ ${ }^{9}$ Observatories of the Carnegie Institution for Science, 813 Santa Barbara St, Pasadena, CA 91101, USA \\ ${ }^{10}$ Spitzer Science Center, MS 314-6, California Institute of Technology, Pasadena, CA 91125, USA \\ ${ }^{11}$ Kavli IPMU (WPI), The University of Tokyo, 5-1-5 Kashiwanoha, Kashiwa-shi, Chiba 277-8583, Japan \\ Received 2014 February 27; accepted 2014 April 15; published 2014 June 3
}

\begin{abstract}
Interaction of supernova (SN) ejecta with the optically thick circumstellar medium (CSM) of a progenitor star can result in a bright, long-lived shock-breakout event. Candidates for such SNe include Type IIn and superluminous $\mathrm{SNe}$. If some of these $\mathrm{SNe}$ are powered by interaction, then there should be a specific relation between their peak luminosity, bolometric light-curve rise time, and shock-breakout velocity. Given that the shock velocity during shock breakout is not measured, we expect a correlation, with a significant spread, between the rise time and the peak luminosity of these SNe. Here, we present a sample of $15 \mathrm{SNe}$ IIn for which we have good constraints on their rise time and peak luminosity from observations obtained using the Palomar Transient Factory. We report on a possible correlation between the $R$-band rise time and peak luminosity of these $\mathrm{SNe}$, with a false-alarm probability of 3\%. Assuming that these $\mathrm{SNe}$ are powered by interaction, combining these observables and theory allows us to deduce lower limits on the shock-breakout velocity. The lower limits on the shock velocity we find are consistent with what is expected for $\mathrm{SNe}$ (i.e., $\sim 10^{4} \mathrm{~km} \mathrm{~s}^{-1}$ ). This supports the suggestion that the early-time light curves of SNe IIn are caused by shock breakout in a dense CSM. We note that such a correlation can arise from other physical mechanisms. Performing such a test on other classes of SNe (e.g., superluminous SNe) can be used to rule out the interaction model for a class of events.
\end{abstract}

Key words: stars: massive - stars: mass-loss - supernovae: general

Online-only material: machine-readable table

\section{INTRODUCTION}

A supernova ( $\mathrm{SN})$ exploding within an optically thick circumstellar medium (CSM) may have several unique characteristics. First, if the Thomson optical depth in the CSM is larger than $c / v_{\mathrm{s}}$, where $c$ is the speed of light and $v_{\mathrm{s}}$ is the shock velocity, then the shock breakout will occur in the CSM rather than near the stellar surface. This will lead to shock-breakout events that are more luminous and longer than those from normal $\mathrm{SNe}$ (e.g., Falk \& Arnett 1977; Ofek et al. 2010; Chevalier \& Irwin 2011; Balberg \& Loeb 2011).

In a CSM with a slowly decreasing radial density profile (e.g., a wind profile with density $\rho \propto r^{-2}$, where $r$ is the radial distance), the radiation-dominated shock will transform to a collisionless shock, generating hard X-ray photons and $\mathrm{TeV}$ neutrinos (Katz et al. 2011; Murase et al. 2011, 2014; Ofek et al. 2013a). While the collisionless shock traverses regions in which the Thomson optical depth, $\tau$, is above a few, the hard X-ray photons can be converted to visible light (e.g., via Comptonization; Chevalier \& Irwin 2012; Svirski et al. 2012). We refer to this as the optically thick interaction phase. In most cases, emission of visible light from the optically thick interaction phase will last on the order of 10 times the shock- breakout timescale (e.g., the time it takes the shock to evolve $\mathrm{e}^{12}$ from $\tau \approx 30$ to $\tau \approx 3$ ). Svirski et al. (2012) showed that the optically thick interaction phase is characterized by bolometric emission with a power-law or broken power-law light curve, with specific power-law indices. A recent example for such behavior was demonstrated by Ofek et al. (2014b) for SN 2010j1 (PTF 10aaxf; see also Moriya et al. 2013; Fransson et al. 2013). However, in most cases the shock-breakout timescale may be less than several days, and the optically thick interaction phase will thus be short and hard to distinguish in the optical band. It is possible that later, when the interaction is moving into the optically thin region, the hard X-ray photons traveling inward toward optically thick regions (e.g., the cold dense shell; Chevalier \& Fransson 1994) will be partially converted to optical photons.

Svirski et al. (2012) and Ofek et al. (2014b) showed that for SNe having light curves that are powered by interaction, there should exist a specific relation between the shock-breakout time scale, the SN luminosity, and the shock velocity at shock breakout. For various reasons the shock velocity is

\footnotetext{
12 In a wind-profile CSM $\left(\rho_{\mathrm{CSM}}=K r^{-2}\right)$ the optical depth is inversely proportional to the radius.
} 
hard to measure. Ignoring the shock velocity will introduce considerable scatter into this relation. However, we still expect a correlation, with a significant spread, between the $\mathrm{SN}$ rise time (i.e., a proxy for the shock-breakout timescale; Ofek et al. 2010) and peak luminosity.

Type IIn SNe (e.g., Filippenko 1997) are characterized by intermediate-width emission lines which are presumably emitted by shock interaction and/or recombination in optically thin gas in the CSM due to the SN radiation field (e.g., Chevalier \& Fransson 1994; Chugai 2001). Furthermore, it was suggested that hydrogen-poor superluminous $\mathrm{SNe}$ are powered by interaction (Quimby et al. 2011; Chevalier \& Irwin 2011; see a review by Gal-Yam 2012), as well as some other rare types of SNe (e.g., Ben-Ami et al. 2014).

Here we perform a simple test of the interaction model for SNe IIn, by searching for a correlation between the rise time and peak luminosity. Indeed, we find a possible correlation between these properties. However, we stress that other models that can produce this correlation cannot yet be ruled out. We present our SN sample and observations in Section 2, and review the predictions in Section 3. The data are analyzed in Section 4, and we discuss the results in Section 5.

\section{SAMPLE AND OBSERVATIONS}

The Palomar Transient Factory (PTF; ${ }^{13}$ Law et al. 2009; Rau et al. 2009) and its extension the intermediate PTF (iPTF) found over 2200 spectroscopically confirmed SNe. We selected 19 SNe IIn for which PTF/iPTF has good coverage of the lightcurve rise and peak; they are listed in Table 1. Optical spectra were obtained with a variety of telescopes and instruments, including the Double Spectrograph (Oke \& Gunn 1982) at the Palomar $5 \mathrm{~m}$ Hale telescope, the Kast spectrograph (Miller \& Stone 1993) at the Lick $3 \mathrm{~m}$ Shane telescope, the Low Resolution Imaging Spectrometer (Oke et al. 1995) on the Keck-1 10 m telescope, and the Deep Extragalactic Imaging Multi-Object Spectrograph (Faber et al. 2003) on the Keck-2 10 m telescope. A representative spectrum of each $\mathrm{SN}$ is available through the WISeREP website ${ }^{14}$ (Yaron \& Gal-Yam 2012).

The PTF/iPTF data were reduced using the IPAC pipeline (Laher et al. 2014). The photometric calibration is described by Ofek et al. (2012a, 2012b). The photometry was performed by running point-spread-function fitting on subtracted images (e.g., Ofek et al. 2013c), and the photometric measurements of all SNe in our sample are listed in Table 2 and shown in Figure 1.

\section{PREDICTIONS}

Next we briefly review the predictions regarding the peak luminosity and rise time in the context of the interaction model. Ofek et al. (2014b) used the Chevalier (1982) selfsimilar hydrodynamical solution describing ejecta with a powerlaw velocity distribution propagating through a CSM with a power-law density distribution. Combining this with the shockbreakout properties and assuming conversion of kinetic energy into luminosity, Ofek et al. predicted a relation of the form

$$
v_{\mathrm{bo}}=t_{\mathrm{bo}}^{(\alpha-1) / 3}\left[2 \pi \epsilon \frac{m-w}{m-3}(w-1) \frac{c}{\kappa L_{0}}\right]^{-1 / 3} .
$$

Here $v_{\text {bo }}$ is the shock-breakout velocity, $t_{\text {bo }}$ is the shock-breakout timescale, $\epsilon$ is the efficiency of converting the kinetic energy to

\footnotetext{
13 http://www.astro.caltech.edu/ptf/

$14 \mathrm{http} / / / \mathrm{www} . w e i z m a n n . a c . i 1 /$ astrophysics/wiserep/
}

luminosity, $\kappa$ is the CSM opacity, $m$ is the power-law index of the ejecta velocity distribution, and $w$ is the negative power-law index of the radial density profile of the CSM (i.e., $\rho=K r^{-w}$ ). We note that for a wind profile CSM $(w=2)$, the mass-loading parameter is $K=\dot{M} /\left(4 \pi v_{\mathrm{w}}\right)$, where $\dot{M}$ is the mass-loss rate and $v_{\mathrm{w}}$ is the CSM velocity. $L_{0}$ is the luminosity extrapolated to a time of $1 \mathrm{~s}$ and is defined by the relations

$$
L=L_{0} t^{\alpha}
$$

and

$$
\alpha=\frac{(2-w)(m-3)+3(w-3)}{m-w} .
$$

To summarize, in interaction-powered $\mathrm{SNe}$ we expect a relation between $t_{\mathrm{bo}}, v_{\mathrm{bo}}$, and $L_{0}$ (Equation (1)). The relevant observables are the rise time, which is a proxy for the shockbreakout timescale (e.g., Ofek et al. 2010), and the peak luminosity, which is a function of $L_{0}$ and $t_{\text {bo. Therefore, we }}$ expect a correlation between the SN peak luminosity and its rise time. However, given the relatively large power-law index in which $v_{\text {bo }}$ appears in Equation (1), relative to those of $t_{\mathrm{bo}}$ and $L_{0}\left(v_{\mathrm{bo}}^{3} \propto t_{\mathrm{bo}}^{\alpha-1} L_{0}\right)$, we predict that this correlation will have a large spread (i.e., the correlation will be weak rather than tight).

\section{ANALYSIS}

In the context of the CSM-shock-breakout model, characterization of the SN rise time requires a model for the functional shape of the rising light curve. Although some progress has been made (e.g., Ginzburg \& Balberg 2014), we still lack such a model. For simplicity, here we fit each SN rising light curve with an exponential function of the form

$$
L=L_{\max }\left\{1-\exp \left[\left(t_{0}-t\right) / t_{\mathrm{e}}\right]\right\}
$$

Here $L$ is the luminosity at time $t$, and the free parameters in the fit are the peak luminosity $L_{\max }$, the time when the flux is zero $t_{0}$, and the characteristic rise time $t_{\mathrm{e}}$. We note that, in our analysis, instead of using the fitted $L_{\max }$, we used the actual maximum observed luminosity. This was done in order to avoid the effect of a possible covariance between $t_{\mathrm{e}}$ and $L_{\max }$ that may arise from the fitting process. The $R$-band luminosity was calculated taking into account the Galactic extinction in the SN direction (Cardelli et al. 1989; Schlegel et al. 1998), the SN luminosity distance (WMAP3 cosmology; Spergel et al. 2007), and assuming that the absolute magnitude of the Sun in the $R_{\text {PTF }}$ band is 4.66 mag (Ofek et al. 2012a).

We also attempted to fit a $t^{2}$ law of the form

$$
L=L_{\max }\left(1-\left[\frac{t-t_{\max }}{\Delta t}\right]^{2}\right)
$$

Here $t_{\max }$ is the maximum of the parabolic fit, and $\Delta t$ is the time from zero to maximum luminosity. In this case, the characteristic rise time (i.e., the time it takes the light curve to rise by a factor of $\exp [1])$ is given by

$$
t_{\mathrm{e}}=\Delta t\left(1+\sqrt{1-e^{-1}}\right) .
$$

Both fits provide a reasonable empirical description of the rising light curves (Figure 2). For the purpose of the analysis presented here, we use the $t_{\mathrm{e}}$ obtained from the exponential fit (Equation (4)). We note that, qualitatively, the results do not change if one uses the rise time obtained from the parabolic fit. 
Table 1

Supernovae Sample

\begin{tabular}{|c|c|c|c|c|c|c|c|c|c|c|c|c|c|}
\hline Name & $\begin{array}{l}\text { R.A. } \\
\text { (deg) }\end{array}$ & $\begin{array}{l}\text { Decl. } \\
\text { (deg) }\end{array}$ & $z$ & $\begin{array}{c}\text { DM } \\
(\mathrm{mag})\end{array}$ & $\begin{array}{l}E_{B-V} \\
\text { (mag) }\end{array}$ & $\begin{array}{c}t_{0} \\
(\mathrm{MJD})\end{array}$ & $\begin{array}{c}t_{\max } \\
(\mathrm{MJD})\end{array}$ & $\begin{array}{c}L_{\max } \\
\left(\mathrm{erg} \mathrm{s}^{-1}\right)\end{array}$ & $\begin{array}{c}L_{0} \\
\left(\text { erg s}^{-1}\right)\end{array}$ & $\begin{array}{c}t_{\mathrm{e}} \\
\text { (day) }\end{array}$ & $\chi^{2} /$ dof & $\begin{array}{c}v_{\text {bo }} \\
\left(\mathrm{km} \mathrm{s}^{-1}\right)\end{array}$ & $\begin{array}{c}\log _{10} K \\
\left(\mathrm{~g} \mathrm{~cm}^{-1}\right)\end{array}$ \\
\hline PTF10cwx & 188.3189 & -0.0530 & 0.073 & 37.58 & 0.025 & 55243.3 & 55275.3 & $7.2 \times 10^{42}$ & $4.7 \times 10^{44}$ & $12.7 \pm 5.7$ & $8.5 / 7$ & 3300 & 17.0 \\
\hline PTF10gvf & 168.4385 & 53.6291 & 0.081 & 37.82 & 0.011 & 55319.9 & 55337.5 & $1.0 \times 10^{43}$ & $5.5 \times 10^{44}$ & $6.6 \pm 1.5$ & $27.4 / 15$ & 4600 & 16.7 \\
\hline PTF10hbf & 193.1944 & -6.9220 & 0.042 & 36.32 & 0.036 & 55292.1 & 55320.6 & $9.9 \times 10^{41}$ & $6.5 \times 10^{43}$ & $13.3 \pm 4.4$ & $3.9 / 9$ & 1700 & 17.1 \\
\hline PTF10oug & 260.1866 & 29.0738 & 0.150 & 39.26 & 0.042 & 55378.5 & 55424.4 & $3.3 \times 10^{43}$ & $2.5 \times 10^{45}$ & $20.9 \pm 5.3$ & $13.8 / 14$ & 4600 & 17.2 \\
\hline PTF10qaf & 353.9287 & 10.7758 & 0.284 & 40.82 & 0.074 & 55350.4 & 55409.4 & $1.7 \times 10^{44}$ & $1.3 \times 10^{46}$ & $25.6 \pm 8.7$ & $0.3 / 4$ & 7400 & 17.3 \\
\hline PTF10tyd & 257.3309 & 27.8191 & 0.063 & 37.25 & 0.065 & 55419.5 & 55470.5 & $5.3 \times 10^{42}$ & $4.0 \times 10^{44}$ & $20.4 \pm 2.0$ & $34.9 / 17$ & 2500 & 17.2 \\
\hline PTF10vag & 326.8270 & 18.1310 & 0.052 & 36.81 & 0.111 & 55445.4 & 55464.9 & $7.0 \times 10^{42}$ & $3.6 \times 10^{44}$ & $5.6 \pm 2.5$ & $16.4 / 4$ & 4300 & 16.7 \\
\hline PTF10weh & 261.7103 & 58.8521 & 0.138 & 39.06 & 0.032 & 55450.3 & 55526.3 & $5.8 \times 10^{43}$ & $5.8 \times 10^{45}$ & $54.8 \pm 12.4$ & $14.8 / 11$ & 4000 & 17.7 \\
\hline PTF10yyc & 69.8221 & -0.3488 & 0.214 & 40.12 & 0.046 & 55476.7 & 55506.8 & $5.1 \times 10^{43}$ & $3.0 \times 10^{45}$ & $9.1 \pm 3.7$ & $26.5 / 14$ & 7100 & 16.9 \\
\hline PTF10achk & 46.4898 & -10.5225 & 0.033 & 35.77 & 0.063 & 55534.3 & 55551.5 & $5.3 \times 10^{42}$ & $2.6 \times 10^{44}$ & $5.0 \pm 1.0$ & $54.8 / 10$ & 4000 & 16.6 \\
\hline PTF11fzz & 167.6945 & 54.1052 & 0.082 & 37.85 & 0.011 & 55723.6 & 55798.6 & $3.3 \times 10^{43}$ & $2.4 \times 10^{45}$ & $18.2 \pm 1.4$ & $121.1 / 19$ & 4800 & 17.2 \\
\hline PTF12cxj & 198.1612 & 46.4851 & 0.036 & 35.96 & 0.011 & 56029.6 & 56050.1 & $2.6 \times 10^{42}$ & $1.5 \times 10^{44}$ & $9.1 \pm 1.0$ & $93.2 / 51$ & 2600 & 16.9 \\
\hline PTF12glz & 238.7210 & 3.5354 & 0.079 & 37.76 & 0.131 & 56107.6 & 56155.4 & $3.3 \times 10^{43}$ & $2.7 \times 10^{45}$ & $26.3 \pm 2.2$ & $42.7 / 33$ & 4300 & 17.3 \\
\hline PTF12ksy & 62.9421 & -12.4669 & 0.031 & 35.66 & 0.043 & 56232.5 & 56256.7 & $5.2 \times 10^{42}$ & $3.7 \times 10^{44}$ & $17.3 \pm 6.4$ & $136.2 / 12$ & 2700 & 17.2 \\
\hline iPTF13agz & 218.6338 & 25.1621 & 0.057 & 37.02 & 0.033 & 56377.5 & 56418.3 & $3.9 \times 10^{42}$ & $2.8 \times 10^{44}$ & $18.0 \pm 2.5$ & $76.7 / 58$ & 2400 & 17.2 \\
\hline PTF09drs & 226.6257 & 60.5943 & 0.045 & 36.49 & 0.017 & 55025.5 & 55066.5 & $5.2 \times 10^{42}$ & $3.7 \times 10^{44}$ & $17.7 \pm 16.3$ & $14.3 / 6$ & 2600 & 17.2 \\
\hline PTF10cwl & 189.0919 & 7.7939 & 0.085 & 37.93 & 0.022 & 55245.1 & 55261.7 & $9.1 \times 10^{42}$ & $4.5 \times 10^{44}$ & $5.0 \pm 12.8$ & $0.6 / 2$ & 4800 & 16.6 \\
\hline PTF10tel & 260.3778 & 48.1298 & 0.035 & 35.93 & 0.016 & 55427.8 & 55442.6 & $7.3 \times 10^{42}$ & $3.9 \times 10^{44}$ & $6.6 \pm 3.5$ & $4.3 / 2$ & 4100 & 16.7 \\
\hline PTF12efc & 224.1447 & 39.6848 & 0.234 & 40.34 & 0.012 & 56052.7 & 56155.5 & $6.8 \times 10^{43}$ & $7.9 \times 10^{45}$ & $88.0 \pm \cdots$ & $18.9 / 19$ & 3700 & 17.9 \\
\hline
\end{tabular}

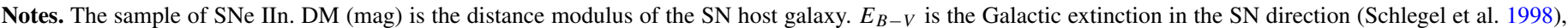

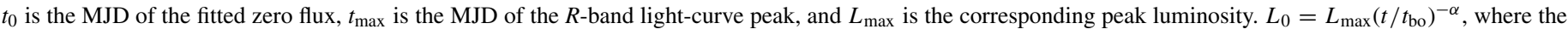

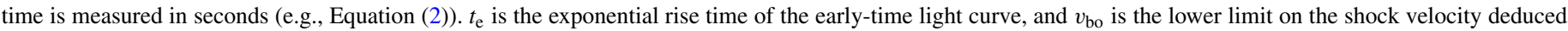

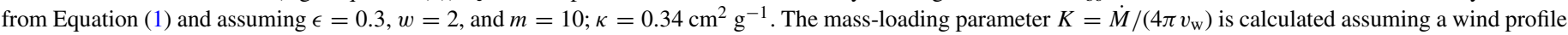

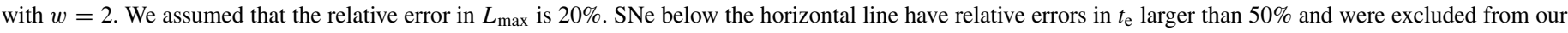



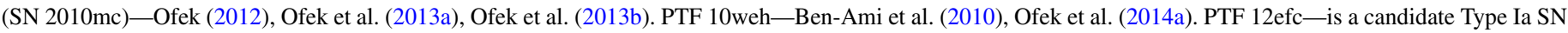

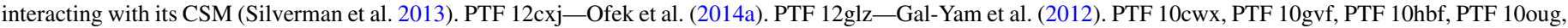

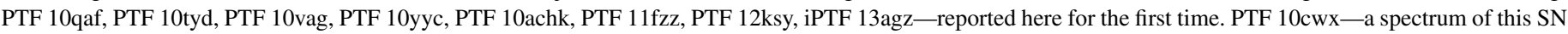

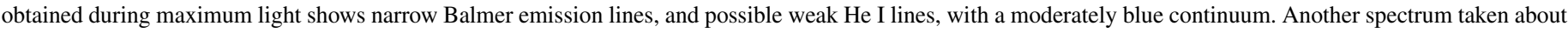



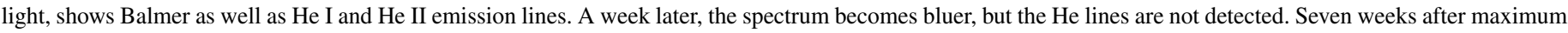

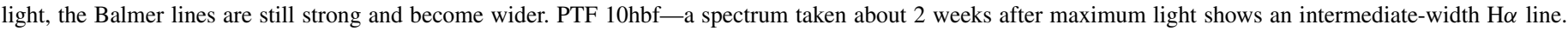

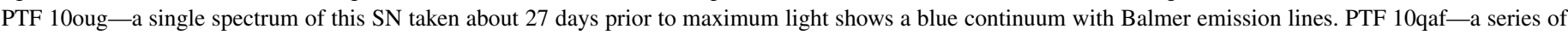

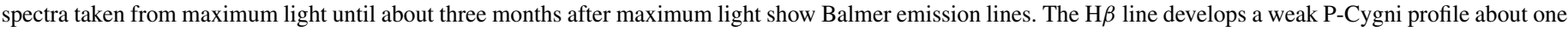

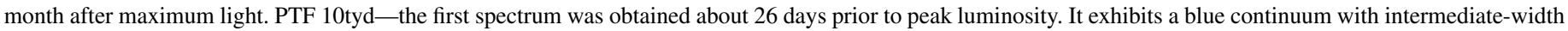

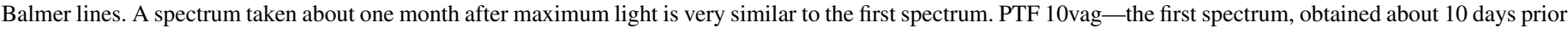

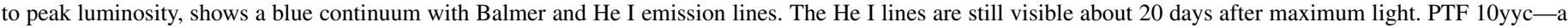

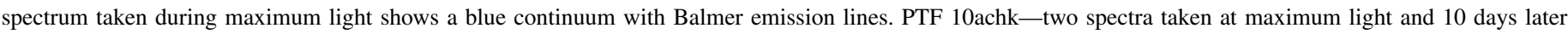

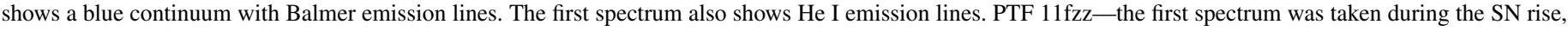

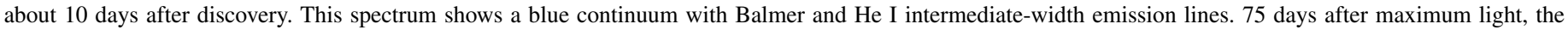




strong P-Cygni profile. iPTF 13agz — a spectrum obtained about one month after maximum light shows a blue continuum with Balmer emission lines.

The best-fit exponential rise time and maximum luminosity for each $\mathrm{SN}$ are listed in Table 1.

For the $19 \mathrm{SNe}$ in our sample, we estimated the errors in the rise times using the bootstrap technique (Efron 1982). For 4 out of 19 events the relative errors are larger than 50\%; they appear in Table 1 below the horizontal line and are marked on the plots with gray boxes. We flagged these events as unreliable and they were not used in the correlation analysis.

Figure 2 presents the observed $L_{\max }$ versus $t_{\mathrm{e}}$. The Spearman rank correlation of the remaining $15 \mathrm{SNe}$ in our sample is 0.49 . Using the bootstrap technique (Efron 1982) we find that the probability to get a correlation coefficient larger than that is 0.03 . Therefore, the correlation is significant at the $2.5 \sigma$ level. We note that the Spearman rank correlation is not sensitive to the distribution of variables, while the use of the bootstrap technique give us an estimate of the false-alarm probability
Table 2

Supernovae Photometry

\begin{tabular}{lccccc}
\hline \hline Name & Telescope & Filter & $\begin{array}{c}\text { MJD } \\
(\text { day })\end{array}$ & $\begin{array}{c}R_{\text {PTF }} \\
(\mathrm{mag})\end{array}$ & $\begin{array}{c}\text { Err } \\
(\mathrm{mag})\end{array}$ \\
\hline PTF12ksy & PTF & $R$ & 56202.486 & 20.414 & 0.143 \\
PTF12ksy & PTF & $R$ & 56235.251 & 19.175 & 0.098 \\
PTF12ksy & PTF & $R$ & 56235.280 & 19.038 & 0.059 \\
PTF12ksy & PTF & $R$ & 56237.255 & 18.840 & 0.057 \\
PTF12ksy & PTF & $R$ & 56237.288 & 18.919 & 0.033 \\
\hline
\end{tabular}

Notes. Photometric measurements of the $\mathrm{SNe}$ in the sample. This table contains measurements from the PTF/iPTF telescope as well as the Palomar 60 inch and Liverpool $2 \mathrm{~m}$ telescope.

(This table is available in its entirety in a machine-readable form in the online journal. A portion is shown here for guidance regarding its form and content.) 



Time since $\mathrm{t}_{0}$ [day]

Figure 1. Light curves of the $19 \mathrm{SNe}$ in our sample, for which we attempted to fit the rise time using the exponential rise function (Equation (4)). The black circles show the PTF/iPTF $R$-band measurements with their uncertainties, while the gray lines represent the best-fit exponential rise function. The SN name is marked in each subplot. The values of $t_{0}$ are listed in Table 1 .

taking into account the scatter in the data, but without using the formal errors in the variables. Furthermore, we tried different statistical approaches that make quite different assumptions and obtained very similar results.

Our results may be affected by selection biases and therefore should be treated with care. A possible selection effect is that $\mathrm{SNe}$ with longer rise times are easier to detect even if they are faint. However, such a selection effect will introduce an anticorrelation between the rise time and peak luminosity. Another concern is if the luminosity span of the light-curve rise can affect our fitting. In order to check for this and other selection effects, we also look for correlations between the luminosity ratio of the first $\mathrm{SN}$ detection and its peak luminosity, and the $\mathrm{SN}$ rise time as well as the $\mathrm{SN}$ peak luminosity. We do not find any evidence for such correlations.

We conclude that there is marginal evidence for a correlation between the rise time and peak luminosity of SNe IIn, and that in this stage we cannot rule out the possibility that these $\mathrm{SNe}$ are powered by interaction.
Next, we use Equation (2), with the constants listed below, to calculate $L_{0}$. Figure 3 shows $L_{0}$ versus $t_{\mathrm{e}} . L_{0}$ is a function of $L_{\max }$ and $t_{\mathrm{e}}$, and therefore Figure 3 shows two nonindependent parameters. However, in Figure 2 we already showed that there is a possible correlation between an independent version of these parameters. We note that in the context of the interaction model $t_{\mathrm{e}}$ is our best estimate for $t_{\mathrm{bo}}$. Most importantly, the power-law index of $v_{\mathrm{bo}}$ in Equation (1) is larger than that of $L_{0}$ and $t_{\mathrm{bo}}$. Therefore, we expect that Figure 3 will exhibit a large scatter.

We stress that theory as well as some UV observations suggest that the bolometric rise time can be faster than the $R$-band rise time (e.g., Roming et al. 2012; Gal-Yam et al. 2014); hence, our $t_{\mathrm{e}}$ is likely only an upper limit on $t_{\mathrm{bo}}$. Moreover, $L_{0}$ was estimated based on the $R$-band magnitude rather than the bolometric magnitudes. Therefore, these $L_{0}$ values should be regarded as lower limits.

Overplotted on Figure 3 are the equal shock-velocity contours, as calculated using Equation (1), assuming $w=2$ (i.e., wind profile), $m=12, \kappa=0.34 \mathrm{~cm}^{2} \mathrm{r}^{-1}$, and $\epsilon=0.3$. These 


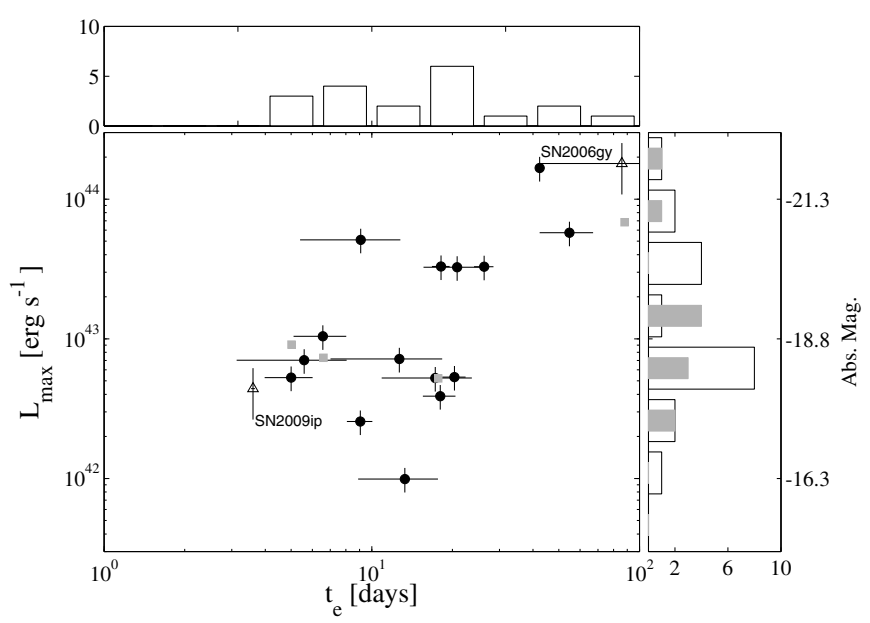

Figure 2. Peak luminosity ( $\left.L_{\max }\right)$ vs. exponential rise $\left(t_{\mathrm{e}}\right)$ for the $\mathrm{SNe}$ in our sample. The black circles are for the SNe whose relative errors on the best-fit exponential rise are smaller than $50 \%$, while the gray squares are for all the other $\mathrm{SNe}$. We did not plot the errors for the gray squares, and the corresponding $\mathrm{SNe}$ were not used in our correlation analysis. The white histograms on the top and right sides present the $t_{\mathrm{e}}$ and $L_{\max }$ distributions, respectively, for all 19 sources. The narrower gray bars on the right histogram show the peak absolute magnitude distribution of 11 SNe IIn discussed by Kiewe et al. (2012). Also shown (empty triangles) are the positions of some other events: SN 2006gy (Ofek et al. 2007, Smith et al. 2007) and SN 2009ip (Prieto et al. 2013, Ofek et al. 2013c, Margutti et al. 2014). The rise time for these SNe was fitted in a way similar to that for the main $\mathrm{SNe}$ in our sample.

values of $m$ and $w$ were also used to calculate $L_{0}$. We note that $m=12$ (10) is expected in the case of a convective (radiative) envelope (Matzner \& McKee 1999), and that the value of $\alpha$ is not very sensitive to the value of $m$ (see Ofek et al. 2014b).

Furthermore, $\epsilon=0.3$ is close to the maximum possible efficiency. Given that our measurements provide an upper limit on $t_{\mathrm{bo}}$ and a lower limit on $L_{0}$, the deduced breakout shock velocities in Figure 3 are only a lower limit on the actual shock velocity at breakout.

\section{DISCUSSION}

There is a growing line of evidence that SNe IIn are embedded in a large amount of CSM ejected months to years prior to their explosions (e.g., Dopita et al. 1984; Weiler et al. 1991; Chugai \& Danziger 1994; Smith et al. 2008; Gal-Yam \& Leonard 2009; Kiewe et al. 2012; Ofek et al. 2013c). In some cases we probably see optical outbursts associated with these mass-loss events (e.g., Foley et al. 2007; Pastorello et al. 2007; Mauerhan et al. 2013; Corsi et al. 2014; Fraser et al. 2013; Ofek et al. 2013c, 2014a). This CSM is likely to be optically thick and lead to luminous and long shock-breakout events (Ofek et al. 2010; Chevalier \& Irwin 2011).

For some SNe the early-time light curve is powered by shock breakout in a dense CSM followed by conversion of the kinetic energy to optical luminosity via shock interaction in optically thick regions. In such cases, Svirski et al. (2012) and Ofek et al. (2014b) predicted a relation between the shock-breakout timescale $\left(t_{\text {bo }}\right)$, velocity $\left(v_{\text {bo }}\right)$, and the SN peak luminosity $L_{\max }$.

Based on a sample of 15 SNe IIn from PTF/iPTF, we show that there is a possible correlation between their rise time and peak luminosity. Interpreting this correlation in the context of the relation predicted by Ofek et al. (2014b), the deduced lower limits on the shock velocity are consistent with the expected shock velocity from $\mathrm{SNe}$ (i.e., on the order of $10^{4} \mathrm{~km} \mathrm{~s}^{-1}$ ). Our findings support the suggestion made by Ofek et al. (2010)

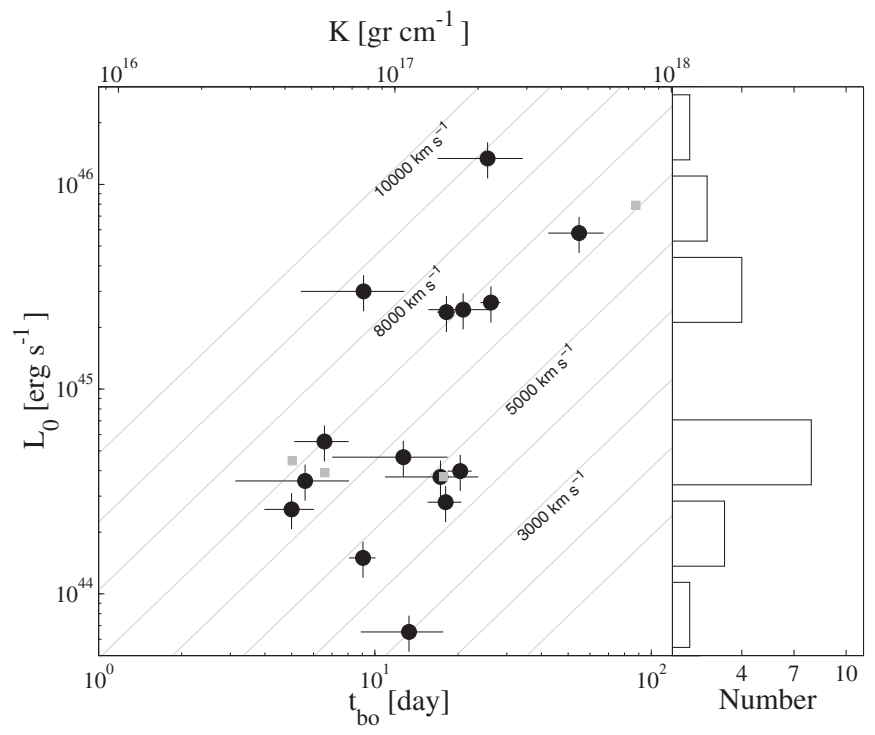

Figure 3. Shock-breakout timescale $\left(t_{\mathrm{bo}}\right)$ vs. $L_{0}$. The shock breakout timescale is assumed to be identical to $t_{\mathrm{e}}$, while $L_{0}$ is calculated from Equation (2). Symbols as in Figure 2. Lines of equal shock-breakout velocity, as calculated using Equation (1), are shown as gray dashed lines. We note that, in the context of the interaction model, our measurements provide a lower limit on $L_{0}$ and $t_{\mathrm{bo}}$; thus, they represent a lower limit on the breakout shock velocity. The upper abscissa gives the mass-loading parameter $K$ assuming a wind profile (i.e., $w=2$; see Equation (8) in Ofek et al. 2014b). The vertical histogram on the right shows the $L_{0}$ distribution for all $19 \mathrm{SNe}$ in our sample.

and Chevalier \& Irwin (2011) that the early-time light curves of some SNe IIn are powered by shock breakout in a dense CSM. However, we note that the light curves may be contaminated by additional sources of energy (e.g., radioactivity), adding additional spread into the expected relation. Furthermore, our observations cannot yet be used to rule out other alternatives (at least not without a detailed model in hand).

In Figure 3 there is a puzzling deficiency of objects around $L_{0} \approx 10^{45} \mathrm{erg} \mathrm{s}^{-1}$, and maybe also some concentration of events with $L_{0} \approx 4 \times 10^{44} \mathrm{erg} \mathrm{s}^{-1}$. We note that comparison of the $L_{\max }$ distribution of our sample and that of $11 \mathrm{SNe}$ IIn reported by Kiewe et al. (2012) suggests that this feature may be caused by small-number statistics (Figure 2). Finally, we propose that application of this test to other classes of SNe can be used to rule out the hypothesis that they are powered by interaction of their ejecta with a dense CSM.

We thank Dan Perley for obtaining some spectra. E.O.O. thanks Ehud Nakar and Orly Gnat for discussions. This paper is based on observations obtained with the Samuel Oschin Telescope as part of the Palomar Transient Factory project, a scientific collaboration between the California Institute of Technology, Columbia University, Las Cumbres Observatory, the Lawrence Berkeley National Laboratory, the National Energy Research Scientific Computing Center, the University of Oxford, and the Weizmann Institute of Science. Some of the data presented herein were obtained at the W. M. Keck Observatory, which is operated as a scientific partnership among the California Institute of Technology, the University of California, and NASA; the Observatory was made possible by the generous financial support of the W. M. Keck Foundation. We are grateful for excellent staff assistance at Palomar, Lick, and Keck Observatories. E.O.O. is incumbent of the Arye Dissentshik career development chair and is grateful to support by grants from 
the Willner Family Leadership Institute Ilan Gluzman (Secaucus NJ), Israeli Ministry of Science, Israel Science Foundation, Minerva, Weizmann-UK and the I-CORE Program of the Planning and Budgeting Committee and The Israel Science Foundation. A.G-Y. acknowledge grants from the ISF, BSF, GIF, Minerva, the EU/FP7 via ERC grant (307260), and the I-CORE program of the Planning and Budgeting Committee and The Israel Science Foundation. M.M.K. acknowledges generous support from the Hubble Fellowship and Carnegie-Princeton Fellowship. A.V.F.'s SN group at UC Berkeley has received generous financial assistance from Gary and Cynthia Bengier, the Christopher R. Redlich Fund, the Richard and Rhoda Goldman Fund, the TABASGO Foundation, and NSF grant AST-1211916.

\section{REFERENCES}

Balberg, S., \& Loeb, A. 2011, MNRAS, 414, 1715

Ben-Ami, S., Badenes, C., Kulkarni, S. R., et al. 2010, ATel, 2961, 1 Ben-Ami, S., Gal-Yam, A., Mazzali, P. A., et al. 2014, ApJ, 785, 37

Cardelli, J. A., Clayton, G. C., \& Mathis, J. S. 1989, ApJ, 345, 245

Chevalier, R. A. 1982, ApJ, 259, 302

Chevalier, R. A., \& Fransson, C. 1994, ApJ, 420, 268

Chevalier, R. A., \& Irwin, C. M. 2011, ApJL, 729, L6

Chevalier, R. A., \& Irwin, C. M. 2012, ApJL, 747, L17

Chugai, N. N. 2001, MNRAS, 326, 1448

Chugai, N. N., \& Danziger, I. J. 1994, MNRAS, 268, 173

Corsi, A., Ofek, E. O., Gal-Yam, A., et al. 2014, ApJ, 782, 42

Dopita, M. A., Cohen, M., Schwartz, R. D., \& Evans, R. 1984, ApJL, 287, L69

Drake, A. J., Djorgovski, S. G., Mahabal, A. A., et al. 2010, ATel, 2555, 1

Efron, B. 1982, The Jackknife, the Bootstrap and Other Resampling Plans

(Philadelphia, PA: The Society for Industrial and Applied Mathematics)

Faber, S. M., Phillips, A. C., Kibrick, R. I., et al. 2003, Proc. SPIE, 4841, 1657

Falk, S. W., \& Arnett, W. D. 1977, ApJS, 33, 515

Filippenko, A. V. 1997, ARA\&A, 35, 309

Foley, R. J., Smith, N., Ganeshalingam, M., et al. 2007, ApJL, 657, L105

Fransson, C., Ergon, M., Challis, P. J., et al. 2013, arXiv:1312.6617

Fraser, M., Magee, M., Kotak, R., et al. 2013, ApJL, 779, L8

Gal-Yam, A. 2012, Sci, 337, 927

Gal-Yam, A., Arcavi, I., Ofek, E. O., et al. 2014, Natur, 509, 471

Gal-Yam, A., Ben-Ami, S., Arcavi, I., et al. 2012, ATel, 4293, 1
Gal-Yam, A., \& Leonard, D. C. 2009, Natur, 458, 865

Ginzburg, S., \& Balberg, S. 2014, ApJ, 780, 18

Katz, B., Sapir, N., \& Waxman, E. 2011, arXiv:1106.1898

Kiewe, M., Gal-Yam, A., Arcavi, I., et al. 2012, ApJ, 744, 10

Laher, R. R., Surace, J., Grillmair, C. J., et al. 2014, arXiv:1404.1953

Law, N. M., Kulkarni, S. R., Dekany, R. G., et al. 2009, PASP, 121, 1395

Margutti, R., Milisavljevic, D., Soderberg, A. M., et al. 2014, ApJ, 780, 21

Matzner, C. D., \& McKee, C. F. 1999, ApJ, 510, 379

Mauerhan, J. C., Smith, N., Filippenko, A., et al. 2013, MNRAS, 430, 1801

Miller, J. S., \& Stone, R. P. S. 1993, Lick Observatory Technical Report 66 (Santa Cruz, CA: Lick Observatory)

Moriya, T. J., Maeda, K., Taddia, F., et al. 2013, MNRAS, 435, 1520

Murase, K., Thompson, T. A., Lacki, B. C., \& Beacom, J. F. 2011, PhRvD, 84,043003

Murase, K., Thompson, T. A., \& Ofek, E. O. 2014, MNRAS, 440, 2528

Ofek, E. O. 2012, CBET, 3313, 1

Ofek, E. O., Cameron, P. B., Kasliwal, M. M., et al. 2007, ApJL, 659, L13

Ofek, E. O., Fox, D., Cenko, S. B., et al. 2013a, ApJ, 763, 42

Ofek, E. O., Laher, R., Law, N., et al. 2012a, PASP, 124, 62

Ofek, E. O., Laher, R., Surace, J., et al. 2012b, PASP, 124, 854

Ofek, E. O., Lin, L., Kouveliotou, C., et al. 2013b, ApJ, 768, 47

Ofek, E. O., Rabinak, I., Neill, J. D., et al. 2010, ApJ, 724, 1396

Ofek, E. O., Sullivan, M., Cenko, S. B., et al. 2013c, Natur, 494, 65

Ofek, E. O., Sullivan, M., Shaviv, N. J., et al. 2014a, arXiv:1401.5468

Ofek, E. O., Zoglauer, A., Boggs, S. E., et al. 2014b, ApJ, 781, 42

Oke, J. B., Cohen, J. G., Carr, M., et al. 1995, PASP, 107, 375

Oke, J. B., \& Gunn, J. E. 1982, PASP, 94, 586

Pastorello, A., Smartt, S. J., Mattila, S., et al. 2007, Natur, 447, 829

Prieto, J. L., Brimacombe, J., Drake, A. J., \& Howerton, S. 2013, ApJL, 763, L27

Quimby, R. M., Kulkarni, S. R., Kasliwal, M. M., et al. 2011, Natur, 474,487

Rau, A., Kulkarni, S. R., Law, N. M., et al. 2009, PASP, 121, 1334

Roming, P. W. A., Pritchard, T. A., Prieto, J. L., et al. 2012, ApJ, 751, 92

Schlegel, D. J., Finkbeiner, D. P., \& Davis, M. 1998, ApJ, 500, 525

Silverman, J. M., Nugent, P. E., Gal-Yam, A., et al. 2013, ApJS, 207, 3

Smith, N., Chornock, R., Li, W., et al. 2008, ApJ, 686, 467

Smith, N., Li, W., Foley, R. J., et al. 2007, ApJ, 666, 1116

Spergel, D. N., Bean, R., Doré, O., et al. 2007, ApJS, 170, 377

Svirski, G., Nakar, E., \& Sari, R. 2012, ApJ, 759, 108

Weiler, K. W., Van Dyk, S. D., Discenna, J. L., Panagia, N., \& Sramek, R. A. 1991, ApJ, 380, 161

Yaron, O., \& Gal-Yam, A. 2012, PASP, 124, 668 